J. Korean Math. Soc. 46 (2009), No. 3, pp. 463-473

DOI 10.4134/JKMS.2009.46.3.463

\title{
FREDHOLM MAPPINGS AND BANACH MANIFOLDS
}

\author{
José María Soriano Arbizu
}

\begin{abstract}
Two $C^{1}$-mappings, whose domain is a connected compact $C^{1}$-Banach manifold modelled over a Banach space $X$ over $\mathbb{K}=\mathbb{R}$ or $\mathbb{C}$ and whose range is a Banach space $Y$ over $\mathbb{K}$, are introduced. Sufficient conditions are given to assert they share only a value. The proof of the result, which is based upon continuation methods, is constructive.
\end{abstract}

\section{Preliminaries}

Scientific phenomena are locally described by parameters, whose choice is sometimes arbitrary. This implies the importance of the availability of a methodology for the comparison of results of measurements. Locally, a Banach manifold looks like a Banach space. For a local description, different Banach (or coordinate or parameter) spaces are allowed and transformation rules exist for these coordinates.

Let $X, Y$ be two Banach spaces. Let $u: U \subset X \rightarrow Y$ be a continuous mapping. One way of solving the equation

$$
u(x)=y
$$

for any fixed $y \in Y$, is to embed (1) in a continuum of problems

$$
H(x, t)=y,(0 \leq t \leq 1),
$$

which is solved when $t=0$. When $t=1$, problem (2) becomes (1). If it is possible to continue the solution for all $t \in[0,1]$, then (1) is solved. This is the continuation method with respect to a parameter [1-25]. A continuation method was introduced to solve (1) when $u: M \rightarrow \mathbb{R}^{n}$, where $M$ is a connected compact $C^{1}$-Banach manifold modelled on $\mathbb{R}^{n}$, and $H(\cdot, \cdot)$ is a $C^{1}$-mapping [25].

Here $M$ is a Banach manifold modelled on an infinite-dimensional Banach space $X$ over $\mathbb{K}=\mathbb{R}$ or $\mathbb{C}$ and $u$ ranges over an infinite-dimensional Banach space $Y$ over $\mathbb{K}$.

Received July 11, 2007.

2000 Mathematics Subject Classification. Primary 58C30, Secondary 65H20.

Key words and phrases. regular value, continuation methods, atlas, chart, Banach manifold, compactness.

This work is partially supported by D.G.E.S. Pb 96-1338-CO 2-01 and the Junta de Andalucia.

(C)2009 The Korean Mathematical Society 
Sufficient conditions are given to prove that two $C^{1}$-mappings, of which one is Fredholm of index zero, share only one value on a Banach manifold by using continuation methods on charts. Other conditions, sufficient to guarantee the existence of zero points, have been given by the author in several other papers [7-25]. This shared value can be estimated following a curve. The proof supplies the existence of a curve which leads to the point whose image is the shared value. The keys are the use of the chart spaces [27], the compactness and connectness of $M$, together with the use of the Continuous Dependence Theorem (Theorem 2) [26] and Theorem 1 [28].

We briefly recall some theorems and notation to be used.

Definitions and Notation. [26-28]. Let $F: D(F): X \rightarrow Y$, where $X, Y$ are Banach spaces over $\mathbb{K}$. If $D(F)$ is open, then mapping $F$ is said to be a Fredholm mapping if and only if both $F$ is a $C^{1}$-mapping and $F^{\prime}(x): X \rightarrow Y$ is a Fredholm linear mapping for all $x \in D(F)$. That $L: X \rightarrow Y$ is a linear Fredholm mapping means that $L$ is linear and continuous and both the numbers $\operatorname{dim}(\operatorname{ker}(L))$ and $\operatorname{codim}(R(L))$ are finite, where dim signifies dimension, codim codimension, ker kernel and $\mathrm{R}(L)$ stands for the range of mapping $L$. Therefore $\operatorname{ker}(L)=X_{1}$ is a Banach space and has topological complement $X_{2}$, since $\operatorname{dim}\left(X_{1}\right)$ is finite. The integer number $\operatorname{ind}(L)=\operatorname{dim}(\operatorname{ker}(L))$-codim $(R(L))$ is called the index of $L$. Let $\mathcal{F}(X, Y)$ denote the set of all linear Fredholm mappings $L: X \rightarrow Y$.

Let $M$ be a topological space. A $\operatorname{chart}(U, \varphi)$ in $M$ is a pair where the set $U$ is open in $M$ and $\varphi: U \rightarrow U_{\varphi}$ is a homeomorphism onto an open subset $U_{\varphi}$ of a Banach space $X_{\varphi}$. We call $\varphi$ a chart map, $X_{\varphi}$ is called chart space, and $U_{\varphi}$ chart image. For $x \in U, x_{\varphi}=\varphi(x)$ is called the representative of $x$ in the chart $(U, \varphi)$ or the local coordinate of $x$ in the local coordinate system $\varphi$. The point $x \in M$ may have different local coordinates $x_{\varphi}=\varphi(x)$ and $x_{\psi}=\psi(x)$ for two different charts $(U, \varphi)$ and $(V, \psi)$, respectively. The transformation rules between them are $x_{\varphi}=\varphi\left(\psi^{-1}\left(x_{\psi}\right)\right)$ and $x_{\psi}=\psi\left(\varphi^{-1}\left(x_{\varphi}\right)\right)$.

Two charts, $(U, \varphi)$ and $(V, \psi)$ in $M$, are called $C^{k}$-compatible if and only if $U \cap V=\emptyset$, or both $\varphi \circ \psi^{-1}: \psi(U \cap V) \rightarrow \varphi(U \cap V)$ and $\psi \circ \varphi^{-1}: \varphi(U \cap V) \rightarrow$ $\psi(U \cap V)$ are $C^{k}$-mappings, $k \geq 0$.

A $C^{k}$-atlas for $M, 0 \leq k \leq \infty$ is a collection of charts $\left(U_{i}, \varphi_{i}\right)$, where $i \in I$, which satisfies the following conditions:

(i) the $U_{i}$ cover $M$,

(ii) any two charts are $C^{k}$-compatible,

(iii) all chart spaces $X_{i}$ are Banach spaces over $\mathbb{K}$.

If there is a $C^{k}$-atlas for $M$, then $M$ is said to be a $C^{k}$-Banach manifold. If all chart spaces are equal to a fixed Banach space $X, \mathrm{M}$ is called a $C^{k}$-Banach manifold modelled on $X$. Here manifolds without boundaries will be considered, such as the surface of a ball in $\mathbb{R}^{n}$, an open set in a Banach space $X$, etc.

Let $M$ and $N$ be $C^{k}$-Banach manifolds with chart spaces over $\mathbb{K}, k \geq 1$. The mapping $f: M \rightarrow N$ is called a $C^{r}$-mapping, where $r \leq k$, if and only if $f$ is $C^{r}$ 
at each point $x \in M$ in fixed admissible charts. This means the following: If $(U, \varphi)$ and $(V, \psi)$ are charts in $M$ and $N$ respectively, with $x \in U$ and $f(x) \in V$, then the mapping $\bar{f}=\psi \circ f \circ \varphi^{-1}$, which is well defined in a sufficiently small neighbourhood of $x_{\varphi}$, is $C^{r}$ in the usual sense. $\bar{f}$ is called a representative of $f$.

Two $C^{1}$-curves in $M$, which pass through the point $x \in M$, are called equivalent the point $x$ if and only if the representatives have the same tangent vector at $x$ in some fixed admissible chart. A tangent vector $v$ (otherwise known as $\left.x^{\prime}\left(t_{0}\right)\right)$ to $M$ at $x$ consists of all $C^{1}$-curves which are equivalent at $x$ to a fixed $C^{1}$-curve. The tangent "abstract" vector $v$ of the previous definition to the curve $x(\cdot): U\left(t_{0}\right) \subset \mathbb{R} \rightarrow M, x=x(t)$ at the point $x\left(t_{0}\right)$, has its representative or local coordinate $v_{\varphi}=x_{\varphi}^{\prime}\left(t_{0}\right)$ in the chart $(U, \varphi)$, where $x_{\varphi}=x_{\varphi}(t)=\varphi(x(t))$.

The tangent space $T M_{x}$ to $M$ at the point $x$ is by definition the set of all tangent vectors. It is proven that this is a topological vector space which is linear homeomorphic to each chart space $X_{\varphi}$ at the point $x$.

The map $f^{\prime}(x): T M_{x} \rightarrow T N_{f(x)}$ is called the tangent map of $f: M \rightarrow N$ at point $x$, which is clearly the normal $F$-derivative in local coordinates.

A mapping $f: M \rightarrow N$ is called a Fredholm operator at $x$ if and only if the linearization $f^{\prime}(x): M_{x} \rightarrow N_{f(x)}$ is a Fredholm operator. Furthermore, $f$ is a Fredholm operators at $x$ if and only if the representatives of $f$ in local charts are Fredholm operator at the corresponding points.

Let $f: M \rightarrow N$ be a $C^{k}$-mapping, $k \geq 1$, where $M$ and $N$ are $C^{k}$-Banach manifolds with chart space over $\mathbb{K}$. The mapping $f$ is called a submersion at $x$ if and only if $f^{\prime}(x)$ is surjective and the null space $\operatorname{ker}\left(f^{\prime}(x)\right)$ splits the tangent space of $M$ at point $x$ (which is automatic when $\operatorname{ker}\left(f^{\prime}(x)\right)=\{0\}$ ). A point $x \in M$ is called a regular point of $f$ if and only if $f$ is a submersion at $x$. A point $y \in N$ is called a regular value of $f$ if and only if the set $f^{-1}(y)$ is empty or consists only of regular points.

If $X, Y$ are Banach spaces, let $\mathcal{L}(X, Y)$ denote the set of all linear continuous mappings $L: X \rightarrow Y$. Let $\operatorname{Isom}(X, Y)$ denote the set of all the linear homeomorphisms $L: X \rightarrow Y$. Let $B\left(x_{0}, \rho\right)$ be the open ball of centre $x_{0}$ and radius $\rho$. If $u: X \rightarrow Y$ is a linear continuous bijective operator, then the inverse linear continuous operator will be denoted by $u^{-1}$.

Mapping $\bar{H}_{x}\left(x_{\varphi}, t\right)$ denotes the partial F-derivative of mapping $\bar{H}$ with respect to $X$ at the point $\left(x_{\varphi}, t\right)$, where $\bar{H}: U_{\varphi} \times[0,1] \subset X \times \mathbb{R} \rightarrow Y$.

A representative point always has its corresponding chart map as subindex.

Theorem 1 ([28], p. 300). If $S \in \mathcal{F}(X, Y)$, where $X, Y$ are Banach spaces over $\mathbb{K}$, then there is a number $\varepsilon>0$ such that

$$
T \in \mathcal{F}(X, Y) \quad \text { and } \quad \text { Ind } T=\text { Ind } S
$$

for all linear Fredholm mappings $T \in \mathcal{L}(X, Y)$ with $\|T-S\|<\varepsilon$.

Theorem 2 (Continuous Dependence Theorem [26], pp. 18-19). Let the following conditions be satisfied: 
(i) $P$ is a metric space, called the parameter space.

(ii) For each parameter $p \in P$, mapping $T_{p}$ satisfies the following hypotheses:

(1) $T_{p}: M \rightarrow M$, i.e. $M$ is mapped into itself by $T_{p}$.

(2) $M$ is a closed non-empty set in a complete metric space $(X, d)$.

(3) $T_{p}$ is $k$-contractive for any fixed $k \in[0,1)$.

(iii) For each $p_{0} \in P$, and any $x \in M, \lim _{p \rightarrow p_{0}} T_{p}(x)=T_{p_{0}}(x)$.

Then for each $p \in P$, the equation $x_{p}=T_{p} x_{p}$ has exactly one solution $x_{p}$, where $x_{p} \in M$ and $\lim _{p \rightarrow p_{0}} x_{p}=x_{p_{0}}$.

\section{Fredholm mappings on compact Banach manifolds}

Theorem 3. Let $f, g: M \rightarrow Y$ be two $C^{1}$-mappings, where $M$ is a compact, connected, $C^{1}$-Banach manifold modelled on $X$, where $X, Y$ are two Banach spaces over $\mathbb{K}=\mathbb{R}$ or $\mathbb{C}$. Let $\left(U_{i}, \varphi_{i}\right)_{i \in I}, I=1,2, \ldots, N$ be a $C^{1}$-atlas for $M$. Suppose that the following conditions hold:

(i) Mapping $f$ has only one zero $x^{\star}$ in $M$, with $x^{*} \in U_{j}$, and $f$ is a Fredholm mapping of index zero at $x^{*}$.

(ii) For any fixed $t \in[0,1]$, zero is a regular value of any mapping

$$
H(\cdot, t): M \times\{t\} \rightarrow Y, \quad H(\cdot, t):=f(\cdot)-t g(\cdot) .
$$

Hence the following statement holds true:

(a) The mappings $f$ and $g$ share only one value $x^{* *}$ on $M$.

(b) There is a $C^{1}$-mapping $\alpha(\cdot):[0,1] \rightarrow M$, with

$$
\begin{gathered}
H(\alpha(t), t)=0, \forall t \in[0,1], \alpha(0)=x^{*}, \alpha(1)=x^{* *}, \text { where } \\
H: M \times[0,1] \rightarrow Y, H(x, t)=f(x)-t g(x) .
\end{gathered}
$$

Proof. $\mathcal{L}(X, Y)$ is provided by the topology given by its operator norm and $X \times \mathbb{R}$ is provided by a product topology.

The point $x^{*}$ belongs to $U_{j}, j \in I$, and $x_{\varphi_{j}}^{*}=\varphi_{j}\left(x^{*}\right)$ is its representative point in the chart $\left(U_{j}, \varphi_{j}\right)$. The representative mapping of the mapping $H$ in this chart $\left(U_{j}, \varphi_{j}\right)$ is the $C^{1}$-mapping

$$
\bar{H}: U_{j_{\varphi j}} \times[0,1] \subset X \times \mathbb{R} \rightarrow Y, \bar{H}\left(x_{\varphi_{j}}, t\right):=\left(H \circ\left(\varphi_{j}^{-1}, I d\right)\right)\left(x_{\varphi_{j}}, t\right),
$$

with $I d(t):=t$. This mapping verifies that $\bar{H}\left(x_{\varphi_{j}}^{*}, 0\right)=0$.

The representative mapping of mapping $H$ on any chart is also written as $\bar{H}$ for simplicity, and the same criterium will be used for any mapping. Any extended mapping will be denoted as the original mapping.

(a) By hypothesis (i), $f$ is a Fredholm mapping of index zero at $x^{*}$, i.e., the representative of $f$ in local charts $\bar{f}$ are Fredholm mappings of index zero at the corresponding representative points. Since $f$ is a $C^{1}$ mapping and since index is an integer, therefore Theorem 1 implies that Ind $f^{\prime}(x)$ is locally constant. 
Hence, since $M$ is connected, $f$ is a Fredholm mapping of index zero on $M$. Hence

$$
f^{\prime}(x) \in \mathcal{F}(X, Y) \text {, and Ind } f^{\prime}(x)=0, \forall x \in M .
$$

Since $H$ is a $C^{1}$ mapping, therefore $\forall \varepsilon>0,\left\|\bar{f}^{\prime}\left(x_{\varphi_{i}}\right)-\left(\bar{f}^{\prime}\left(x_{\varphi_{i}}\right)-t \bar{g}^{\prime}\left(x_{\varphi_{i}}\right)\right)\right\|=|t|\left\|\bar{g}^{\prime}\left(x_{\varphi_{i}}\right)\right\|<\varepsilon$, when $|t|<\delta(\varepsilon)$, and since the index is an integer and $[0,1]$ is connected, Theorem 1 and Equation (3) imply for any fixed $x \in M$ in local charts, that

$$
\bar{f}^{\prime}\left(x_{\varphi_{i}}\right)-t \bar{g}^{\prime}\left(x_{\varphi_{i}}\right) \in \mathcal{F}(X, Y)
$$

and

$$
\operatorname{Ind}\left(\bar{f}^{\prime}\left(x_{\varphi_{i}}\right)-t \bar{g}^{\prime}\left(x_{\varphi_{i}}\right)\right)=0, \forall i \in I, \forall t \in[0,1] .
$$

Hence for any fixed $t \in[0,1], H(\cdot, t)$ is a Fredholm mapping and $\operatorname{Ind}\left(H_{x}(x, t)\right)=$ $\operatorname{Ind}\left(f^{\prime}(x)\right)=0$, and

$$
\operatorname{Ind}\left(\bar{H}_{x}\left(x_{\varphi_{i}}, t\right)\right)=0, i \in I, x \in U_{i}, t \in[0,1] .
$$

By hypothesis (ii), since zero is a regular value of the mapping $H(\cdot, t)$ for any fixed $t \in[0,1]$, therefore the mapping

$$
\bar{H}_{x}\left(x_{\varphi_{i}}, t\right)(\cdot) \in \mathcal{L}(X, Y)
$$

maps $X$ onto $Y$ for any fixed $\left(x_{\varphi_{i}}, t\right) \in\left(U_{\varphi_{i}} \times[0,1]\right) \cap \bar{H}^{-1}(0)$, therefore

$$
\operatorname{codim}\left(\operatorname{range}\left(\bar{H}_{x}\left(x_{\varphi_{i}}, t\right)\right)\right)=0,
$$

and hence

$$
\operatorname{Ind}\left(\bar{H}_{x}\left(x_{\varphi_{i}}, t\right)\right)=\operatorname{dim}\left(\operatorname{ker}\left(\bar{H}_{x}\left(x_{\varphi_{i}}, t\right)\right)\right) .
$$

Equations (4) and (5) imply that

$$
\operatorname{Ind}\left(\bar{H}_{x}\left(x_{\varphi_{i}}, t\right)\right)=0=\operatorname{dim}\left(\operatorname{ker}\left(\bar{H}_{x}\left(x_{\varphi_{i}}, t\right)\right)\right),
$$

i.e., $\left(\bar{H}_{x}\left(x_{\varphi_{i}}, t\right)\right)$ is injective. Therefore $\bar{H}_{x}\left(x_{\varphi_{i}}, t\right)(\cdot)$ is a linear continuous bijective mapping, and since $X, Y$ are Banach spaces, the linear mapping $\bar{H}_{x}\left(x_{\varphi_{i}}, t\right)^{-1}(\cdot)$ is continuous. Hence $\bar{H}_{x}\left(x_{\varphi_{i}}, t\right)(\cdot)$ is a linear homeomorphism, i.e.,

$$
\bar{H}_{x}\left(x_{\varphi_{i}}, t\right)(\cdot) \in \operatorname{Isom}(X, Y) .
$$

(b) Let us fix any chart of the atlas $\left(U_{j}, \varphi_{j}\right), j \in I$, which will be called $(U, \varphi)$, whose corresponding chart image is $U_{\varphi}$. Let us suppose that $\left(x_{a}, t_{a}\right) \in$ $H^{-1}(0)$ with $x_{a} \in U$. Such a point $\left(x_{a}, t_{a}\right)$ will be call a "starting point".

The representative mapping of $H$ in the chart $(U, \varphi)$

$$
\bar{H}: U_{\varphi} \times[0,1] \subset X \times \mathbb{R} \rightarrow Y,
$$

clearly has as zero $\left(x_{a_{\varphi}}, t_{a}\right)$ with $x_{a_{\varphi},}=\varphi\left(x_{a}\right)$.

The representative point of $\left(x_{a}, t_{a}\right)$ in the chart $(U \times[0,1],(\varphi, I d))$ verifies $\left(x_{a_{\varphi}}, t_{a}\right) \in \bar{H}^{-1}(0) \cap\left(U_{\varphi} \times[0,1]\right)$. Such a point $\left(x_{a_{\varphi}}, t_{a}\right)$ will be called a 
"representative starting point" in $U_{\varphi} \times[0,1]$, and there is a positive number $R$ such that the ball $B\left(x_{a_{\varphi}}, R\right) \subset U_{\varphi}$, and furthermore

$$
\left\|\bar{H}_{x}\left(x_{a_{\varphi}}, t_{a}\right)^{-1}\right\|=C .
$$

(b1) Two positive numbers $r_{a}, r_{0_{a}}$ must be found for later use.

Let us construct the set

$$
B=B\left(x_{a_{\varphi}}, R\right) \times[0,1]
$$

and define the mapping

$$
\begin{gathered}
\bar{h}: B \subset U_{\varphi} \times \mathbb{R} \rightarrow Y, \\
\bar{h}\left(\left(x_{a_{\varphi}}+x_{\varphi}\right), t\right):=\bar{H}_{x}\left(x_{a_{\varphi}}, t_{a}\right)\left(\left(x_{a_{\varphi}}+x_{\varphi}\right)-x_{a_{\varphi}}\right)-\bar{H}\left(\left(x_{a_{\varphi}}+x_{\varphi}\right), t\right) .
\end{gathered}
$$

Since $\bar{h}$ is a continuous mapping as a composition of continuous mappings, therefore for any $r>0$ and for $C$ given above, there is a

$$
\delta\left(\frac{r}{2 C}\right)>0
$$

such that, if $\left(\left(x_{a_{\varphi}}+x_{\varphi}\right), t\right), \in B$ with

$$
\begin{gathered}
\left\|x_{\varphi}, t-t_{a}\right\|<\delta\left(\frac{r}{2 C}\right) \text { then } \\
\left\|\bar{h}\left(\left(x_{a_{\varphi}}+x_{\varphi}\right), t\right)-\bar{h}\left(x_{a_{\varphi}}, t_{a}\right)\right\|<\frac{r}{2 C} .
\end{gathered}
$$

On the other hand, another mapping can be defined as

$$
\begin{aligned}
\bar{h}_{x} & : B \rightarrow \mathcal{L}(X, Y) \\
\bar{h}_{x}\left(\left(x_{a_{\varphi}}+x_{\varphi}\right), t\right) & :=\bar{H}_{x}\left(x_{a_{\varphi}}, t_{a}\right)-\bar{H}_{x}\left(x_{a_{\varphi}}+x_{\varphi}, t_{a}\right)
\end{aligned}
$$

and is also continuous in the set $B$, and therefore there is an

$$
r:=\delta\left(\frac{1}{2 C}\right)>0
$$

such that, if $\left(\left(x_{a_{\varphi}}+x_{\varphi}\right), t\right) \in B$ with

$$
\begin{gathered}
\left\|\left(x_{\varphi}, t-t_{a}\right)\right\|<\delta\left(\frac{1}{2 C}\right), \text { then } \\
\left\|\bar{h}_{x}\left(\left(x_{a_{\varphi}}+x_{\varphi}\right), t\right)-\bar{h}_{x}\left(x_{a_{\varphi}}, t_{a}\right)\right\|<\frac{1}{2 C} .
\end{gathered}
$$

By taking $r$ given by Equation (8) and fixing $r_{0}^{\prime}:=\delta\left(\frac{r}{2 C}\right)$, given by Equation (6), the number $r_{0}:=\min \left\{r, r_{0}^{\prime}\right\}$ can be defined. We select $r_{a}:=\min \{R, r\}$ and $r_{0_{a}}=\min \left\{R, r_{0}\right\}$.

(b2) The sets

$$
I_{a}:=\left\{t \in[0,1]:\left|t-t_{a}\right| \leq r_{0 a}\right\}, A_{a}:=\left\{x_{\varphi} \in X:\left\|x_{\varphi}\right\| \leq r_{a}\right\},
$$


will be associated to the "representative starting point" $\left(x_{a_{\varphi}}, t_{a}\right)$. Since $\left\|x_{\varphi}\right\| \leq R, \forall x_{\varphi} \in A_{a}$, therefore

$$
\left(x_{\varphi}+x_{a_{\varphi}}\right) \in U_{\varphi} \subset X_{\varphi} .
$$

Given a "starting point" $\left(x_{a}, t_{a}\right)$ and its "representative starting point" $\left(x_{a_{\varphi}}\right.$, $\left.t_{a}\right)$, the existence of two continuous mappings are proved:

$\bar{\alpha}(\cdot): I_{a} \subset \mathbb{R} \rightarrow A_{a}+x_{a_{\varphi}} \subset U_{\varphi} \subset X$, such that $\bar{H}(\bar{\alpha}(t), t)=0, \forall t \in I_{a}$

and

such that

$$
\alpha(\cdot): I_{a} \subset \mathbb{R} \rightarrow U \subset M
$$

$$
H(\alpha(t), t)=0, \forall t \in I_{a} .
$$

Let us solve the equation

$$
\bar{H}\left(\left(x_{a_{\varphi}}+x_{\varphi}\right), t\right)=0,
$$

for fixed $t \in I_{a}$ when $x_{\varphi}$ is in $A_{a}$. Obviously, $\bar{H}\left(x_{a_{\varphi}}, t_{a}\right)=0$. Equation (10) is equivalent to the following equation

$$
\bar{H}_{x}\left(x_{a_{\varphi}}, t_{a}\right)^{-1}\left[\bar{H}_{x}\left(x_{a_{\varphi}}, t_{a}\right)\left(x_{\varphi}\right)-\bar{H}\left(\left(x_{a_{\varphi}}+x_{\varphi}\right), t\right)\right]=x_{\varphi},
$$

which leads us to define the mappings

$$
\begin{aligned}
\bar{h}_{t} & : A_{a} \times\{t\} \rightarrow Y \text { for fixed } t \in I_{a}, \\
\bar{h}_{t}\left(x_{\varphi}\right) & :=\bar{H}_{x}\left(x_{a_{\varphi}}, t_{a}\right)\left(x_{\varphi}\right)-\bar{H}\left(\left(x_{a_{\varphi}}+x_{\varphi}\right), t\right)=\bar{h}\left(\left(x_{a_{\varphi}}+x_{\varphi}\right), t\right),
\end{aligned}
$$

and

$$
\bar{T}_{t}: A_{a} \rightarrow X, \bar{T}_{t}\left(x_{\varphi}\right):=\bar{H}_{x}\left(x_{a_{\varphi}}, t_{a}\right)^{-1} \bar{h}_{t}\left(x_{\varphi}\right) .
$$

Observe that $\bar{h}_{t}\left(x_{\varphi}\right)$ is $\bar{h}\left(\left(x_{a_{\varphi}}+x_{\varphi}\right), t\right)$ defined in (b1) when $t$ is fixed and belongs to $I_{a}$. Let us also observe that $t$ in the definitions of $\bar{h}_{t}$ and $\bar{T}_{t}$ is an index to highlight that $t$ is fixed.

Evidently

$$
\bar{h}_{t_{a}}(0)=0
$$

$$
\bar{h}_{t_{a}}^{\prime}(0)=0 \text {. }
$$

Equation (11) is equivalent to the following key Fixed Point Equation

$$
\bar{T}_{t}\left(x_{\varphi}\right)=x_{\varphi},
$$

which is studied below.

Let $x_{\varphi}, x_{\varphi}^{\prime} \in A_{a}, t \in I_{a}$, and hence the Taylor Theorem together with Equations (9) and (13) imply that

$$
\begin{aligned}
& \left\|\bar{h}_{t}\left(x_{\varphi}\right)-\bar{h}_{t}\left(x_{\varphi}^{\prime}\right)\right\| \\
\leq & \sup \left\{\left\|\bar{h}_{t}^{\prime}\left(x_{\varphi}^{\prime}+\theta\left(x_{\varphi}-x_{\varphi}^{\prime}\right)\right)\right\|: \theta \in[0,1]\right\} \cdot\left\|x_{\varphi}-x_{\varphi}^{\prime}\right\| \leq \frac{1}{2 C} r_{a} .
\end{aligned}
$$


Equations (12) and (15) imply that

$$
\left\|\bar{h}_{t}\left(x_{\varphi}\right)\right\| \leq\left\|\bar{h}_{t}\left(x_{\varphi}\right)-\bar{h}_{t_{a}}(0)\right\|+\left\|\bar{h}_{t_{a}}(0)\right\| \leq \frac{r_{a}}{2 C}
$$

hence

$$
\left\|\bar{T}_{t}\left(x_{\varphi}\right)\right\| \leq\left\|\bar{H}_{x}\left(x_{a_{\varphi}}, t_{a}\right)^{-1}\right\|\left\|\bar{h}\left(x_{\varphi}, t\right)\right\| \leq r_{a} .
$$

We will apply Theorem 2 to the sets and mappings which have just been defined. The metric space $\left(I_{a},|\cdot|\right)$ is the parameter space of hypothesis (i) needed in Theorem 2. The set $A_{a}$ is considered as the closed and non-empty set and $X$ as the complete metric space of hypothesis (ii), which is verified below:

From Equation (16), for any fixed $t \in I_{a}$, and for all $x_{\varphi} \in A_{a}$, we have $\left\|\bar{T}_{t} x_{\varphi}\right\| \leq r_{a}$, therefore $\bar{T}_{t} x_{\varphi} \in A_{a}$, and hence $\bar{T}_{t}: A_{a} \rightarrow A_{a}$, i.e., $\bar{T}_{t}$ maps the closed and non-empty set $A_{a}$ of the Banach space $X$ into itself.

From Equations (9), (13), and the Taylor Theorem, for any $x_{\varphi}, x_{\varphi}^{\prime} \in A_{a}$, and for any $t \in I_{a}$, the following holds:

$$
\begin{aligned}
\left\|\bar{T}_{t}\left(x_{\varphi}\right)-\bar{T}_{t}\left(x_{\varphi}^{\prime}\right)\right\| & \leq\left\|\bar{H}_{x}\left(x_{a_{\varphi}}, t_{a}\right)^{-1}\right\|\left\|\bar{h}_{t}^{\prime}\left(x_{\varphi}^{\prime}+\theta\left(x_{\varphi}-x_{\varphi}^{\prime}\right)\right)\right\|\left\|x_{\varphi}-x_{\varphi}^{\prime}\right\| \\
& \leq C\left\|\bar{h}_{t}^{\prime}\left(x_{\varphi}^{\prime}+\theta\left(x_{\varphi}-x_{\varphi}^{\prime}\right)\right)-\bar{h}_{t_{a}}^{\prime}(0)\right\|\left\|x_{\varphi}-x_{\varphi}^{\prime}\right\| \\
& \leq \frac{1}{2}\left\|x_{\varphi}-x_{\varphi}^{\prime}\right\|,(\theta \in[0,1]) .
\end{aligned}
$$

Therefore $\bar{T}_{t}$ is half-contractive for any fixed $t \in I_{a}$. Hence hypothesis (ii) of Theorem 2 is verified.

For any fixed $t_{0} \in I_{a}$ and for all $x_{\varphi} \in A_{a}$, the following holds:

$$
\begin{aligned}
\bar{T}_{t}\left(x_{\varphi}\right) & =\bar{H}_{x}\left(x_{a_{\varphi}}, t_{a}\right)^{-1}\left(\bar{H}_{x}\left(x_{a_{\varphi}}, t_{a}\right)\left(x_{\varphi}\right)-\bar{H}\left(x_{a_{\varphi}}+x_{\varphi}, t\right)\right) \\
& \rightarrow \bar{H}_{x}\left(x_{a_{\varphi}}, t_{a}\right)^{-1}\left(\bar{H}_{x}\left(x_{a_{\varphi}}, t_{a}\right)\left(x_{\varphi}\right)-\bar{H}\left(x_{a_{\varphi}}+x_{\varphi}, t_{0}\right)\right) \\
& =\bar{T}_{t_{0}}\left(x_{\varphi}\right) \text { as } t \rightarrow t_{0}, t \in I_{a},
\end{aligned}
$$

therefore hypothesis (iii) of Theorem 2 is also verified. Hence Theorem 2 implies, for any $t \in I_{a}$, that $\bar{T}_{t}$ has a unique fixed point $x_{\varphi} \in A_{a}, \bar{T}_{t}\left(x_{\varphi}\right)=x_{\varphi}:=$ $x_{\varphi}(t)$, and $x_{\varphi}(t) \rightarrow x_{\varphi}\left(t_{0}\right)$ while $t \rightarrow t_{0} ; t, t_{0} \in I_{a}$, i.e., $x_{\varphi}(\cdot)$ is a continuous mapping. Thus for any $t \in I_{a}$ there is only one $x_{\varphi} \in A_{a}$, i.e.,

$$
\bar{T}_{t}\left(x_{\varphi}\right)=x_{\varphi}:=x_{\varphi}(t)
$$

and the mapping, we have just defined, $x_{\varphi}(\cdot)$ verifies

$$
x_{\varphi}(t) \rightarrow x_{\varphi}\left(t_{0}\right), \text { while } t \rightarrow t_{0}, \forall t, t_{0} \in I_{a},
$$

which implies that $x_{\varphi}(\cdot)$ is a continuous mapping. Thus for any $t \in I_{a}$ there is one $x_{\varphi}(t)$ such that

$$
\bar{H}\left(x_{a_{\varphi}}+x_{\varphi}(t), t\right)=0
$$

and furthermore

$$
\bar{H}\left(x_{a_{\varphi}}+x_{\varphi}(t), t\right) \rightarrow \bar{H}\left(x_{a_{\varphi}}+x_{\varphi}\left(t_{0}\right), t_{0}\right)=0 \text { while } t \rightarrow t_{0}, t, t_{0} \in I_{a} .
$$


Let us observe that $\bar{T}_{t_{a}}(0)=0, x_{\varphi}\left(t_{a}\right)=0$.

Equation (17) can be written as $\bar{H}(\bar{\alpha}(t), t)=0$, which is verified for all $t \in I_{a}$, where $\bar{\alpha}$ is the following curve on the chart space $(U, \varphi)$,

$$
\bar{\alpha}: I_{a} \rightarrow U_{\varphi} \subset X, \bar{\alpha}(t):=x_{a_{\varphi}}+x_{\varphi}(t), \text { where } \bar{\alpha}\left(t_{a}\right)=x_{a_{\varphi}},
$$

which is one of the goals of this section.

The continuity of both $\bar{\alpha}$ and $\varphi^{-1}$ lets us construct the following curve $\alpha$ on the topological space $M$.

$$
\alpha: I_{a} \subset \mathbb{R} \rightarrow U \subset M, \alpha(t):=\left(\varphi^{-1} \circ \bar{\alpha}\right) .
$$

Equations (18) and (19) implies that

$$
\alpha\left(t_{a}\right)=x_{a}
$$

to be used in the next section.

Equation (17) implies that

$$
\bar{H}(\bar{\alpha}(t), t)=\left(H \circ\left(\varphi^{-1}, I d\right)\right)(\bar{\alpha}(t), t)=H(\alpha(t), t)=0, \forall t \in I a,
$$

which is the another goal of this section.

(c) Conclusions (a) and (b) will be proved here.

Since $f^{-1}(0)=x^{*}$ from hypothesis (i), then there exists $U_{j}$ such that $H^{-1}(0) \cap\left(U_{j} \times[0,1]\right) \neq \emptyset$, therefore there is a point $\left(x^{*}, 0\right) \in H^{-1}(0), x^{*} \in U_{j}$, i.e., $\left(x^{*}, 0\right)$ is a "starting point".

Since $\left(x_{\varphi_{j}}^{*}, 0\right) \in \bar{H}^{-1}(0) \cap\left(U_{\varphi_{j}} \times[0,1]\right)$, i.e., $\left(x_{\varphi_{j}}^{*}, 0\right)$ is a "representative starting point", therefore from (b2) there exist a set $I_{0}$, and two continuous mappings $\bar{\alpha}$ and $\alpha$ such that

$$
\bar{\alpha}: I_{0} \rightarrow U_{\varphi_{j}} \subset X, \text { which verifies } \bar{H}(\bar{\alpha}(t), t)=0, \forall t \in I_{0},
$$

and

$$
\alpha: I_{0} \rightarrow U_{j} \subset M \text {, which verifies } H(\alpha(t), t)=0, \forall t \in I_{0} .
$$

We want to extend the continuous mapping $\alpha: I_{0} \rightarrow M$ to be a continuous mapping $\alpha:[0,1] \rightarrow M$, and to extend Equation (21) to become

$$
H(\alpha(t), t)=0, \forall t \in[0,1] .
$$

Let us suppose that $\alpha(t) \in M, \forall t \in[0, b], b \in U_{i}$. Mapping $\alpha$ is extended to the right of $b$ by taking $(\alpha(b), b)$, which belongs to $H^{-1}(0) \cap\left(U_{i} \times[0,1]\right), i \in I$, as the following "starting point". Equation (20) enables the continuous extension of the continuous mapping $\alpha$ to the right. The continuous extended mapping is also known as $\alpha$.

Mapping $\alpha$ is successively extended to the right in the same way by using its representative in the different charts of the atlas. Now we consider all intervals 
$[0, b]=I_{\alpha}$ such that $H(\alpha(t), t)=0$, has a solution, $\forall t \in I_{\alpha}$. This solution is unique with maximal interval of existence equal to

$$
J=\bigcup_{\alpha} I_{\alpha}
$$

Since $M$ is compact if $J=[0, b), b<1$, it follows from the compactness of $M$ that $\alpha(t) \rightarrow x \in M$, while $t \rightarrow b^{-}$, with $x \in U_{j}, j \in I$. From the continuity of $H$, we know that $H(x, b)=0$, therefore the point $(x, b) \in U_{j} \times[0,1]$, is a "starting point" and the point $\left(x_{\varphi_{j}}, b\right)$ is a "representative starting point" in $U_{j_{i}} \times[0,1]$ and thus the solution of $H(\alpha(t), t)=0$ can be continued beyond $b$, which is a contradiction. Hence we can continue $\alpha$ until $t=1$, which leads to

$$
H(\alpha(1), 1)=f(\alpha(1))-g(\alpha(1))=0 .
$$

This provides an $x^{* *}$ such that $f\left(x^{* *}\right)=g\left(x^{* *}\right)$ is reached by the continuous mapping $\alpha:[0,1] \rightarrow M$, with $H(\alpha(t), t)=0, \forall t \in[0,1]$.

If $f$ and $g$ shared more than one value, then the previous construction from two different zeros of $f-g$, would lead us to conclude that there are two zeros for $f$, which contradicts one of the hypotheses, and therefore it can be concluded that there is only one single shared value.

\section{References}

[1] J. C. Alexander and J. A. York, The homotopy continuation method: numerically implementable topological procedures, Trans. Amer. Math. Soc. 242 (1978), 271-284.

[2] E. L. Allgower, A survey of homotopy methods for smooth mappings, Numerical solution of nonlinear equations (Bremen, 1980), pp. 1-29, Lecture Notes in Math., 878, Springer, Berlin-New York, 1981.

[3] E. L. Allgower and K. Georg, Numerical Continuation Methods, An introduction. Springer Series in Computational Mathematics, 13. Springer-Verlag, Berlin, 1990.

[4] E. L. Allgower, K. Glashoff, and H. Peitgen, Proceedings of the Conference on Numerical Solution of Nonlinear Equations, Bremen, July 1980, Lecture Notes in Math. 878. Springer-Verlag, Berlin, 1981.

[5] C. B. García and T. I. Li, On the number of solutions to polynomial systems of equations, SIAM J. Numer. Anal. 17 (1980), no. 4, 540-546.

[6] C. B. Garcia and W. I. Zangwill, Determining all solutions to certain systems of nonlinear equations, Math. Oper. Res. 4 (1979), no. 1, 1-14.

[7] J. M. Soriano, Existence of zeros for bounded perturbations of proper mappings, Appl. Math. Comput. 99 (1999), no. 2-3, 255-259.

[8] - Global minimum point of a convex function, Appl. Math. Comput. 55 (1993), no. 2-3, 213-218.

[9] Extremum points of a convex function, Appl. Math. Comput. 66 (1994), no. $2-3,261-266$.

[10] On the existence of zero points, Appl. Math. Comput. 79 (1996), no. 1, 99-104.

[11] On the number of zeros of a mapping, Appl. Math. Comput. 88 (1997), no. 2-3, 287-291.

[12] - On the Bézout theorem real case, Comm. Appl. Nonlinear Anal. 2 (1995), no. $4,59-66$.

[13] - On the Bezout theorem, Comm. Appl. Nonlinear Anal. 4 (1997), no. 2, 59-66.

[14] _ Mappings sharing a value on finite-dimensional spaces, Appl. Math. Comput. 121 (2001), no. 2-3, 391-395. 
[15] _ Compact mappings and proper mappings between Banach spaces that share a value, Math. Balkanica (N.S.) 14 (2000), no. 1-2, 161-166.

[16] Z Z Z Z _ ofos compact perturbations of proper mappings, Comm. Appl. Nonlinear Anal. 7 (2000), no. 4, 31-37.

[17] _ A compactness condition, Appl. Math. Comput. 124 (2001), no. 3, 397-402.

[18] — Open trajectories, Appl. Math. Comput. 124 (2001), no. 2, 235-240.

[19] On the existence of zero points of a continuous function, Acta Math. Sci. Ser. B Engl. Ed. 22 (2002), no. 2, 171-177.

[20] no. $6,682-686$.

[21] _ A stable solution, Appl. Math. Comput. 140 (2003), no. 2-3, 223-229.

[22] Stable and unstable stationary trajectories, Appl. Math. Mech. 26 (2005), no. $1,52-57$.

[23] _ A regular value of compact deformation, Appl. Math. Mech. 27 (2006), no. 9, $1265-1274$.

[24] _ Existence and computation of zeros of perturbed mappings, Appl. Math. Comput. 173 (2006), no. 1, 457-467.

[25] _ Continuation methods in Banach manifolds, Bull. Braz. Math. Soc. (N.S.) 38 (2007), no. 1, 67-80.

[26] E. Zeidler, Nonlinear Functional Analysis and Its Applications. III, Variational methods and optimization. Translated from the German by Leo F. Boron. Springer-Verlag, New York, 1985.

[27] , Nonlinear Functional Analysis and Its Applications. IV, Applications to mathematical physics. Translated from the German and with a preface by Juergen Quandt. Springer-Verlag, New York, 1988.

[28] _ Applied Functional Analysis, Main principles and their applications. Applied Mathematical Sciences, 109. Springer-Verlag, New York, 1995.

Departamento de Análisis Matemático, Facultad de Matemáticas UNIVERSIDAD DE SEVILla

Aptdo. 1160, SEvilla 41080, Spain

E-mail address: soriano@us.es 